Revista PSICOLOGIA, 2014, Vol. 28 (1), 33-38

\title{
Adaptação para uma população de estudantes universitários portugueses da escala de auto-estima de estado de Heatherton e Polivy
}

\author{
Miguel Ramos ${ }^{1}$ \\ ${ }^{1}$ Instituto Universitário de Lisboa (CIS/ISCTE-IUL)
}

Resumo: A auto-estima é um importante conceito usado em diversas áreas da psicologia. Os estudos em português têm usado traduções de escalas de auto-estima geral (ex., a escala de auto-estima de Rosenberg, 1979), mas têm negligenciado importantes aspectos deste constructo como a auto-estima de estado. A auto-estima de estado é fundamental para estudos que apresentem manipulações dado que mede alterações momentâneas da auto-estima. Para colmatar esta lacuna, no presente trabalho procedeu-se a uma adaptação para português da escala multidimensional de auto-estima de estado de Heatherton e Polivy (1991). Os resultados mostraram uma medida fidedigna e com a estrutura factorial consistente com a da escala original, distinguindo os componentes de auto-estima de performance, social, e aparência física. A discussão centra-se sobre as vantagens do uso de uma escala de auto-estima de estado.

Palavras-chave: Auto-estima; Auto-estima de estado; Medição; Escala multidimensional

Portuguese adaptation for university students of Heatherton and Polivy's state self-esteem scale. Self-esteem is a crucial concept for a wide range of areas in psychology. Several studies conducted in portuguese have adapted scales of general self-esteem (e.g., Rosenberg, 1979's self-esteem scale) but have neglected other crucial aspects of this construct such as state self-esteem. To address this limitation, the present article provides a portuguese adaptation of Heatherton and Polivy's (1991) state self-esteem scale. Results suggested a reliable measure with a factorial structure identical to the original scale, which included the components of performance, social, and appearance self-esteem. The discussion focuses on the advantages of using a state self-esteem measure.

Keywords: Self-esteem; State self-esteem; Measurement; Multidimensional scale.

A auto-estima é um conceito central para a Psicologia. Uma procura pela palavra "auto-estima" no motor de busca da ISI Web of Knowledge revela um total de aproximadamente cinquenta mil artigos publicados. Estes artigos abrangem um grande leque de áreas em psicologia como por exemplo estudos de aculturação (Berry \& Sabatier, 2010), comportamento de risco (Backer-Fulghum, Patock-Peckham, King, Roufa, \& Hagen, 2012) ou métodos de acompanhamento psicológico (Morton, Roach, Reid, \& Stewart, 2012). De facto, a partir de finais dos anos 70 registou-se um crescimento de trabalho científico a investigar a forma como as pessoas se vêem a si mesmas. Foi com artigos como os publicados por Kuiper e Rogers (1979) ou Markus (1977) que a auto-estima começou a ser considerada como um constructo fundamental para o estudo da personalidade (para um resumo ver, Swann \& Seyle, 2005).

Esta evolução foi naturalmente acompanhada por avanços na forma de medir a auto-estima. Apesar da existência de diversas escalas e métodos, hoje em dia uma das medidas mais utilizadas é a escala de auto-estima geral de Rosenberg (1979). Esta escala tem sido utilizada sistematicamente em mais de 45 mil artigos segundo a ISI Web of Knowledge, abrangendo diversas áreas e tendo como objectivo medir a auto-estima enquanto traço de personalidade. No entanto, uma outra linha de investigação defende que o auto-conceito ${ }^{2}$ tem características estáveis (e portanto medidas através da escala de Rosenberg) mas também outras características maleáveis e adaptativas (Markus \& Kunda,

Dados de contacto para correspondência: Miguel R. Ramos, CIS-IUL, Av. das Forças Armadas, 1649-026 Lisboa, Portugal. E-mail: mrrst@iscte.pt

Esta investigação foi financiada pela Fundação para a Ciência e a Tecnologia (PTDC/PSI-PSO/098110/2008).

${ }^{2}$ Neste artigo seguem-se as definições de auto-conceito e auto-estima apontadas por Campbell (1990). O auto-conceito passa pelo processo cognitivo de organizar memórias concretas e abstractas sobre o self. É no fundo a informação e percepção que o indivíduo tem sobre si mesmo. A auto-estima passa por um processo avaliativo do self e constitui uma dimensão mais afectiva deste. Entendese, portanto, que o self é constituído por uma componente cognitiva (auto-conceito) e outra afectiva (auto-estima). Para Campbell (1990) estes dois conceitos constituem componentes diferentes do self, mas estão associados. 
1986) às quais as escalas de auto-estima geral não são sensíveis. De certa forma, o auto-conceito pode sofrer alterações momentâneas como por exemplo acontece quando um indivíduo se compara com alguém extremamente bem-sucedido. Nestes casos é possível sentir-se um certo contraste que inevitavelmente se traduz numa redução da auto-estima (Tesser, 1988).

Foi com a ideia de medir estas flutuações na auto-estima que Heatherton e Polivy (1991) desenvolveram uma medida de auto-estima de estado (SSES, State Self-Esteem Scale). Esta medida tem sido bastante usada em psicologia especificamente para situações em laboratório com o objectivo de estudar os efeitos de diferentes condições experimentais na auto-estima (ver por exemplo, Leary, Tambor, Terdal, \& Downs, 1995; Melwani \& Barsade, 2011; Twenge, Baumeister, DeWall, Ciarocco, \& Bartels, 2007). Na investigação em português, diversos estudos têm medido a auto-estima usando adaptações da escala de Rosenberg (por exemplo, Pedro \& Peixoto, 2006; Santos \& Maia, 2003) mas até ao momento não tem havido trabalhos a usar uma escala que seja sensível à fluidez e flutuações do autoconceito. Por até agora não existir uma escala adaptada para português que capture estas características da auto-estima e, também, por ser uma escala fundamental para estudos experimentais, o presente artigo apresenta uma adaptação para uma população de estudantes universitários portugueses da SSES de Heatherton e Polivy (1991).

\section{A AUTO-ESTIMA DE ESTADO}

De acordo com Heatherton e Polivy (1991), a ideia de que a auto-estima está sujeita a flutuações momentâneas vem desde as primeiras reflexões em psicologia. James (1890), definiu a auto-estima como um conceito estável e independente de resultados que possam contrariar o auto-conceito. No entanto, este autor também reconhece que a auto-estima possa ser flexível e susceptível a mudanças conforme os objectivos e as experiências vividas pelas pessoas. Portanto, a auto-estima pode ser considerada como um traço de personalidade estável mas que ao mesmo tempo é maleável de acordo com mudanças contextuais.

A ideia de que a auto-estima é maleável e sujeita a diferenças momentâneas é suportada por uma série de estudos em psicologia. Por exemplo, Savin-Williams e Demo (1983) demonstraram que a autoestima flutua em torno de um auto-conceito mais estável. Ou seja, a auto-estima é um conceito estável em torno do qual se registam mudanças momentâneas impulsionadas por variáveis contextuais. Esta ideia é suportada por diversos estudos mostrando que a forma como um indivíduo se avalia a si mesmo pode mudar em função do contexto (Crocker \& Major, 1989; Markus \& Kunda, 1986; Rosenberg, 1986). De forma geral, apesar de não se esperarem mudanças extremas na auto-estima, a literatura mostra que a auto-estima pode ser momentaneamente alterada em relação ao seu estado habitual e mais estável.

Foi com o intuito de formar uma escala que fosse sensível a pequenas flutuações na auto-estima que Heatherton e Polivy desenvolveram a SSES. Para gerar os itens da sua escala, os autores basearam-se na Janis and Field Scale (JFS; Janis \& Field, 1959). A JFS tem como objectivo medir a auto-estima de uma forma multidimensional focando-se em específico nos componentes de auto-avaliação, capacidade académica, e confiança a nível social. Como a JFS mede uma auto-estima estável e mais ligada à personalidade, foram também acrescentados alguns itens de versões modificadas da JFS de Pliner, Chaiken, e Flett (1990) e Fleming e Courtney (1984).

No total, a SSES é constituída por vinte itens focando-se em três subescalas da auto-estima (performance, social, e aparência) que estão associadas a diferentes conceitos. Por exemplo, a subescala de performance mede até que ponto os indivíduos consideram que a sua performance é desejável. Heatherton e Polivy (1991) mostraram que a auto-estima de performance foi sensível a uma manipulação dos resultados de uma tarefa em laboratório. Ou seja, os participantes que foram induzidos a pensar que tinham falhado a tarefa tiveram resultados significativamente mais baixos do que os restantes. Por outro lado, a subescala de auto-estima social está correlacionada com consciência e ansiedade social, medindo até que ponto as pessoas se sentem mais preocupadas com a sua imagem. Por fim, a aparência está ligada a uma auto-avaliação sobre as características físicas do indivíduo. Nos estudos originais de Heartherton e Polivy (1991) as escalas de aparência e auto-estima social foram sensíveis a um programa de âmbito clínico com o objectivo de melhorar a forma como os indivíduos vêem a sua aparência física e competência social (ver Estudo 5). Os resultados foram no sentido de que os indivíduos que participaram no programa tiveram valores mais elevados nestas duas subescalas da auto-estima quando comparados com um grupo de controlo. Em suma, Heatherton e Polivy (1991) mostraram que as três subescalas são sensíveis a flutuações na auto-estima tanto em tarefas de laboratório como em contexto natural.

De especial importância foi o facto de que as três subescalas terem sido afectadas por diferentes situações, o que salienta a relevância de se usar as subescalas de acordo com diversos problemas e condições experimentais. Os autores recomendam que a subescala de performance seja mais indicada para a realização de tarefas e respectivo feedback em laboratório. A subescala de auto-estima social 
poderá ter uma maior utilidade em estudos sobre imagem ou como uma pessoa se apresenta diante dos outros. Por último, a subescala da auto-estima de aparência poderá ser mais útil em estudos centrados no aspecto físico como, por exemplo, a obesidade.

\section{O PRESENTE ESTUDO}

Esta investigação centrou-se na escala de Heatherton e Polivy (1991), por diversas razões. Primeiro, como inicialmente foi referido, a auto-estima de estado é de extrema relevância para estudos realizados em laboratório ou mesmo em contexto natural em que se introduzam condições experimentais. Só através de uma escala sensível a flutuações momentâneas da auto-estima será possível detectar efeitos das manipulações experimentais. Segundo, a SSES tem a vantagem de ser uma medida multidimensional em que diversas subescalas podem ser usadas consoante os objectivos do investigador. As três subescalas abordam diferentes componentes da auto-estima que podem ser manuseados de forma a servir melhor os objectivos e problemas de investigação. Por fim, é uma escala que desde a sua publicação tem sido revalidada e utilizada em inúmeros tópicos e estudos em psicologia.

\section{MÉTODO}

\section{Participantes}

Este estudo inclui uma amostra com 999 participantes. A amostra foi composta por 572 estudantes universitários do sexo masculino e 426 do sexo feminino ( 1 foi não identificado). A sua idade variou entre os 17 e os 60 anos $(M=22.04, S D=5.19)$. Os participantes voluntariaram-se para este estudo e foram informados de que poderiam desistir a qualquer momento caso desejassem. Foram, também, informados sobre o anonimato e confidencialidade do mesmo.

\section{Material}

Três investigadores com conhecimentos das duas línguas realizaram traduções independentes da escala de inglês para português. As traduções foram comparadas e resultaram na versão final apresentada neste artigo (ver em Anexo). Um item que foi de difícil tradução por introduzir um conceito que não existe em português foi o item original "I feel self-conscious" que foi traduzido para "Sinto-me preocupado com o facto dos outros poderem estar a pensar "mal" de mim". Apesar de não transmitir exactamente a mesma ideia do item em inglês, na versão traduzida manteve-se o carácter de preocupação sobre o meio social que é característico do original e também da subescala da dimensão social na qual está incluído.

\section{Procedimento}

A versão traduzida da SSES foi aplicada em questionários de papel-e-lápis e também online. No início da folha do questionário, os participantes leram uma introdução traduzida do original "Abaixo estão uma série de frases sobre o que está a pensar neste momento. Responda, por favor, de acordo com o que é correcto para si neste preciso momento". Os participantes responderam em escalas entre 1 "discordo completamente" e 7 "concordo completamente", sendo que um valor mais alto indica um maior grau de auto-estima ${ }^{3}$.

\section{RESULTADOS}

\section{Análise Descritiva e Fidelidade}

A Tabela 1 indica os alfas de Cronbach, médias, desvios-padrão e correlações entre as várias subescalas. Em relação à fidelidade, uma análise dos alfas de Cronbach indica que todas as subescalas tiveram uma elevada consistência interna, variando entre .78 (performance) e .85 (social). Em relação às médias, a performance foi a componente com valores mais altos $(M=5.33)$, enquanto a social foi a que teve um valor mais baixo $(M=4.49)$. Tal como na escala original, as correlações entre as subescalas são positivas e significativas (valores entre .46 e $.54 ; p s<.001$ ).

Tabela 1. Coeficientes de fidelidade, médias, desvios-padrão e correlações das três subsescalas da SSES

\begin{tabular}{lcccc}
\hline Dimensão & $\boldsymbol{\alpha}$ & $\boldsymbol{M}(\boldsymbol{D P})$ & $\mathbf{1}$ & $\mathbf{2}$ \\
\hline 1. Performance & .78 & $5.33(0.98)$ & - & \\
2. Social & .85 & $4.49(1.28)$ & $.50^{* *}$ & - \\
3. Aparência & .81 & $4.98(1.20)$ & $.54^{* *}$ & $.46^{* *}$ \\
\hline
\end{tabular}

Notas: $* * p<.001$

\footnotetext{
3 O estudo aqui apresentado reúne os dados de diversos estudos em que a escala foi aplicada como parte de questionários diferentes. Estes questionários tinham como objectivo abordar questões relacionadas com a auto-estima e percepções de justiça. Agregámos os dados porque não se espera que haja uma diferença factorial e das propriedades da SSES.
} 


\section{Análise Factorial}

Para a análise factorial foram seguidos os procedimentos recomendados por Tabachnik e Fidell (2001) e o critério usado para eliminar itens na análise foi o seguinte: (i) itens com um peso factorial abaixo de 0.32; (ii) itens com um peso factorial entre .32 e .39 tendo um peso factorial de $>.20$ noutro(s) factor(es); (iii) itens com um peso factorial entre .40 e .49 tendo um peso factorial de > .30 noutro(s) factor(es); e (iv) itens que não fazem sentido teórico dentro do seu factor.

Procedeu-se a uma análise factorial dos eixos principais com rotação oblíqua (ver Tabela 2). Foi usada uma rotação oblíqua porque em termos teóricos (e práticos também) as três subescalas estão fortemente correlacionadas. Esta análise mostrou três factores que explicam $55 \%$ da variância total.

Tabela 2. Pesos factoriais dos 20 itens de acordo com três factores (Factor 1 = auto-estima performance; Factor 2 = auto-estima social; Factor 3 = auto-estima de aparência).

\begin{tabular}{|c|c|c|c|}
\hline Items & 1 & 2 & 3 \\
\hline \multicolumn{4}{|l|}{ Auto-estima performance } \\
\hline 1 - Sinto-me confiante sobre as minhas capacidades & .536 & -.189 & -.287 \\
\hline 2 - Sinto-me frustrado(a) com o meu desempenho (R) & .507 & .243 & -.023 \\
\hline 3 - Sinto que tenho problemas em compreender o que leio (R) & .502 & .102 & .190 \\
\hline 4 - Sinto-me tão inteligente como os outros & .458 & -.112 & -.127 \\
\hline 5 - Sinto-me confiante de que compreendo as coisas & .679 & -.203 & -.071 \\
\hline 6 - Sinto que neste momento tenho menos capacidades intelectuais do que os outros (R) & .701 & .125 & .051 \\
\hline 7 - Sinto que as coisas não me estão a correr bem (R) & .500 & .224 & -.079 \\
\hline \multicolumn{4}{|l|}{ Auto-estima social } \\
\hline 8 - Estou preocupado(a) se possa ser visto como um sucesso ou fracasso (R) & .015 & .537 & .024 \\
\hline 9 - Sinto-me preocupado com o facto dos outros poderem estar a pensar "mal" de mim (R) & -.014 & .779 & -.115 \\
\hline 10 - Sinto-me descontente comigo mesmo(a) (R) & .451 & .286 & -.272 \\
\hline 11 - Estou preocupado(a) com o que as outras pessoas pensam sobre mim (R) & -.019 & .826 & -.073 \\
\hline 12 - Sinto-me inferior às outras pessoas neste momento (R) & .603 & .262 & -.086 \\
\hline 13 - Estou preocupado(a) com a impressão que estou a transmitir (R) & .032 & .705 & -.060 \\
\hline 14 - Estou preocupado(a) que eu possa parecer "tonto(a)" (R) & .346 & .515 & -.013 \\
\hline \multicolumn{4}{|l|}{ Auto-estima aparência } \\
\hline 15 - Sinto-me satisfeito(a) com o aspecto do meu corpo & -.025 & -.025 & -.866 \\
\hline 16 - Sinto que sou respeitado(a) e admirado(a) pelos outros & .367 & -.176 & -.307 \\
\hline 17 - Sinto-me insatisfeito(a) com o meu peso (R) & -.080 & .204 & -.454 \\
\hline 18 - Sinto-me bem comigo mesmo(a) & .398 & .029 & -.513 \\
\hline 19 - Sinto-me satisfeito(a) com a minha aparência neste momento & .081 & .009 & -.873 \\
\hline 20 - Sinto-me pouco atraente (R) & .199 & .264 & -.453 \\
\hline
\end{tabular}

O Factor 1 (auto-estima de performance) explicou $34.49 \%$ da variância e inclui itens como: "Sintome confiante sobre as minhas capacidades". 0 Factor 2 (auto-estima social) explicou 12.84\% da variância e incluiu itens como: "Sinto-me descontente comigo mesmo(a) (recodificado). Por fim, o Factor 3 (autoestima de aparência) explicou $8.21 \%$ da variância e inclui itens como: "Sinto-me satisfeito(a) com o aspecto do meu corpo". Em termos gerais, todos os itens tiveram pesos factoriais de acordo com os factores da escala original tirando os itens da auto-estima social "Sinto-me inferior às outras pessoas neste momento" e "Sinto-me descontente comigo mesmo(a)" e o item da auto-estima de aparência "Sinto que sou respeitado(a) e admirado(a) pelos outros" que tiveram um elevado peso factorial no Factor 1 (performance). Ao se apagar os três itens fica-se com uma estrutura factorial idêntica à da escala original e mantêm-se os elevados níveis de fidelidade para a subescala de auto-estima social $(\alpha=.84)$ e aparência $(\alpha=.82)$.

\section{DISCUSSÃo}

O trabalho de investigação realizado em Portugal tem estudado e incluído escalas de auto-estima que analisam este conceito enquanto traço de personalidade. 0 problema destas escalas é que são pouco sensíveis a flutuações momentâneas como as verificadas em estudos de laboratório ou experimentais. De forma a colmatar esta lacuna, o estudo presente teve como objectivo adaptar a escala de auto-estima de estado de Heatherton e Polivy (1991) para a língua portuguesa.

Os resultados obtidos no presente estudo foram consistentes com os dos autores da escala original. A análise factorial dos itens traduzidos para português confirmou a estrutura factorial de Heartherton e Polivy (1991). Desta forma, verificou-se que dos 20 itens originais, 17 tiveram um peso factorial de acordo com o suposto teórico nas subescalas de auto-estima de performance, social, e aparência. Estes resultados foram acompanhados por coeficientes de fidelidade elevados para as três subescalas, assim como correlações altas entre estas.

Um item de difícil tradução foi o item "I feel self-conscious" que se traduziu por "Sinto-me preocupado com o facto dos outros poderem estar a pensar "mal" de mim". Apesar de transmitir uma 
ideia um pouco diferente do original, neste item manteve-se o carácter de preocupação sobre os outros e a envolvente social. Apesar destas diferenças, a estrutura factorial apresentada neste estudo demonstrou que o item em português manteve as características da subescala de auto-estima social à semelhança do original.

A tradução apresentada neste artigo apresenta várias vantagens para investigadores que planeiem realizar estudos em português. Primeiro, a tradução da SSES contribui para uma maior consciencialização das diferenças entre medidas de auto-estima e apresenta uma solução mais indicada para trabalhos de laboratório ou de campo que introduzam manipulações. Outra vantagem importante é o facto de a SSES estar dividida em três subescalas explorando diferentes componentes da auto-estima. A disponibilidade de se medir a auto-estima ligada à performance, componente social, ou aparência é uma ferramenta importante para investigadores que queiram formular hipóteses mais específicas e testar efeitos centrados em aspectos particulares da auto-estima.

No entanto, apesar das vantagens apontadas, este estudo contém três limitações importantes. A primeira prende-se com o facto de não se ter conseguido replicar a estrutura factorial na íntegra devido a três itens terem pesos factoriais em factores diferentes dos esperados na escala original. Os itens "Sintome inferior às outras pessoas neste momento" e "Sinto-me descontente comigo mesmo(a)" da autoestima social foram os únicos itens desta subescala a serem eliminados. Uma das possíveis razões relaciona-se com o facto de serem os únicos itens da sua subescala que não mencionam uma preocupação sobre os outros e o meio social. Talvez devido aos contextos em que foi aplicado o presente estudo, sentirse "inferior" ou "descontente" tenha sido interpretado do ponto de vista da performance (factor em que estes itens tiveram um peso factorial elevado). Uma explicação semelhante também pode ser avançada para o único item a ser eliminado da subescala de aparência "Sinto que sou respeitado(a) e admirado(a) pelos outros". Este é o único item da sua subescala que não salienta aspectos físicos e, como tal, pode também ter sido entendido em termos de performance, tal como indicou a análise factorial. Uma segunda limitação está ligada ao facto de se ter inserido a SSES em estudos com outros objectivos e variáveis que podem ter afectado as respostas à escala. Por fim, a tradução foi aplicada a uma amostra de estudantes universitários o que traz algumas limitações ao nível da generalização dos resultados do estudo. Apesar destas três limitações, a tradução deste artigo oferece uma escala com 17 itens e coeficientes de fidelidade elevados para as três subescalas. Apesar também de a amostra ser composta unicamente por estudantes universitários, os itens replicaram a estrutura factorial original obtida com amostras que incluíam diversos grupos sociais e faixas etárias.

Em termos de investigação futura, seria interessante usar a escala aqui apresentada conjuntamente com outras teoricamente associadas à auto-estima de forma a se poder testar as suas correlações e a validade convergente-discriminante.

\section{Conclusão}

Apresentou-se neste estudo uma versão traduzida para português da escala de auto-estima de estado de Heatherton e Polivy (1991). Os resultados foram consistentes com os dos autores da escala permitindo uma validação para o contexto de investigação em português. Acredita-se que este contributo possa ter importantes implicações não só teóricas mas também na forma como são colocados os problemas de investigação. Por fim, acredita-se, também, que esta investigação possa contribuir para um maior foco sobre o que significa a auto-estima e sobre diversos métodos de a medir.

\section{Referências}

Backer-Fulghum, L. M., Patock-Peckham, J. A., King, K. M., Roufa, L., \& Hagen, L. (2012). The stressresponse dampening hypothesis: How self-esteem and stress act as mechanisms between negative parental bonds and alcohol-related problems in emerging adulthood. Addictive Behaviours, 37, 477484.

Berry, J. W. \& Sabatier, C. (2010). Acculturation, discrimination, and adaptation among second generation immigrant youth in Montreal and Paris. International Journal of Intercultural Relations, 34, 191207.

Campbell, J. D. (1990). Self-esteem and clarity of the self-concept. Journal of Personality and Social Psychology, 59, 538-549.

Crocker, J., \& Major, B. (1989). Social stigma and self-esteem: The self-protective properties of stigma. Psychological Review, 96, 608-630.

Fleming, J. S., \& Courtney, B. E. (1984). The dimensionality of self-esteem: II. Hierarchical facet model for revised measurement scales. Journal of Personality and Social Psychology, 46, 404-421.

Heatherton, T. F., Polivy, J. (1991). Development and validation of a scale for measuring state self-esteem. Journal of Personality and Social Psychology, 60, 895-910. 
James, W (1890). Principles of psychology, Volume 1. New York: Henry Holt.

Janis, I. L., \& Field, P. B. (1959). Sex differences and factors related to persuasibility. In C. I. Hovland \& I. L. Janis (Eds.), Personality and persuasibility (pp. 55-68). New Haven, CT: Yale University Press.

Kuiper, N. A., \& Rogers, T. B. (1979). Encoding of personal information: Self- other differences. Journal of Personality and Social Psychology, 37, 499-514.

Leary, M. R., Tambor, E. S., Terdal, S. K., \& Downs, D. L. (1995). Selfesteem as an interpersonal monitor: The sociometer hypothesis. Journal of Personality and Social Psychology, 68, 518-530.

Markus, H. (1977). Self-schemas and processing information about the self. Journal of Personality and Social Psychology, 35, 63-78.

Markus, H., \& Kunda, Z. (1986). Stability and malleability of the selfconcept. Journal of Personality and Social Psychology, 51, 858-866.

Melwani, S., \& Barsade, S. G. (2011). Held in contempt: The psychological, interpersonal, and performance consequences of contempt in a work context. Journal of Personality and Social Psychology, 101, 503520.

Morton, L., Roach, L., Reid, H., \& Stewart, S. H. (2012). An evaluation of a CBT group for women with low self-esteem. Behavioural and Cognitive Psychotherapy, 40, 221-225.

Pedro, N., \& Peixoto, F. (2006). Safisfação professional e auto-estima em professors dos 2. e 3.o ciclos do Ensino Básico. Análise Psicológica, 24, 247-262.

Pliner, P., Chaiken, S., \& Flett, G. L. (1990). Gender differences in concern with body weight and physical appearance over the life span. Personality and Social Psychology Bulletin, 16, 263-273.

Rosenberg, R. (1979). Conceiving the self. New York: Basic Books.

Rosenberg, M. (1986). Self-concept from middle childhood through adolescence. In J. Suls \& A. G. Greenwald (Eds.), Psychological perspectives on the self (Vol. 3, pp. 107-135). Hillsdale, NJ: Erlbaum

Santos, P. J., \& Maia, J. (2003). Análise factorial confirmatória e validação preliminar de uma versão portuguesa da escala de auto-estima de Rosenberg. Psicologia: Teoria, Investigação e Práctica, 2, 253-268.

Savin-Williams, R. G, \& Demo, P. (1983). Situational and transitional determinants of adolescent selffeelings. Journal of Personality and Social Psychology, 44, 820-833.

Swann, W. B., Jr., \& Seyle, C. (2005). Personality psychology's comeback and its emerging symbiosis with social psychology. Personality and Social Psychology Bulletin, 31, 155-165.

Tabachnik, B., \& Fidell, L. S. (2001). Using multivariate statistics. Needham Heights Allyn \& Bacon.

Tesser, A. (1988). Toward a self-evaluation maintenance model of social behavior. Advances in Experimental Social Psychology, 21,181-227.

Twenge, J. M., Baumeister, R., DeWall, C. N., Ciarocco, N., \& Bartels, J. M. (2007). Social exclusion decreases prosocial behavior. Journal of Personality and Social Psychology, 92, 56-66.

Historial do artigo

Recebido $\quad 13 / 03 / 2012$

Aceite $\quad 17 / 09 / 2012$

Publicado $\quad 06 / 2014$

\section{Anexo}

Abaixo estão uma série de frases sobre o que está a pensar neste momento. Responda, por favor, de acordo com o que é correcto para si neste preciso momento.

Auto-estima Performance:

1 - Sinto-me confiante sobre as minhas capacidades

2 - Sinto-me frustrado(a) com o meu desempenho (R)

3 - Sinto que tenho problemas em compreender o que leio (R)

4 - Sinto-me tão inteligente como os outros

5 - Sinto-me confiante de que compreendo as coisas

6 - Sinto que neste momento tenho menos capacidades intelectuais do que os outros (R)

7 - Sinto que as coisas não me estão a correr bem (R)

Auto-estima Social:

8 - Estou preocupado(a) se possa ser visto como um sucesso ou fracasso (R)

9 - Sinto-me preocupado com o facto dos outros poderem estar a pensar "mal" de mim (R)

10 - Estou preocupado(a) com o que as outras pessoas pensam sobre mim (R) 
11 - Estou preocupado(a) com a impressão que estou a transmitir (R)

12 - Estou preocupado(a) que eu possa parecer "tonto(a)" (R)

Auto-estima Aparência:

13 - Sinto-me satisfeito(a) com o aspecto do meu corpo

14 - Sinto-me insatisfeito(a) com o meu peso (R)

15 - Sinto-me bem comigo mesmo(a)

16 - Sinto-me satisfeito(a) com a minha aparência neste momento

17 - Sinto-me pouco atraente (R) 